\title{
CRYPTATE INCLUSION COMPLEXES, EFFECTS ON SOLUTE-SOLUTE AND SOLUTE-SOLVENT INTERACTIONS AND ON IONIC REACTIVITY
}

\author{
J.M. LEHN \\ Institut Le Bel, Université Louis Pasteur, 4 Rue Blaise Pascal, 67000 \\ Strasbourg and Collège de France, 11 Place Marcelin Berthelot, 75005 \\ Paris, France.
}

\begin{abstract}
Cryptation of a suitable metal cation by inclusion into a macrobicyclic ligand leads to a large organic cation presenting much weaker interactions with the environment. As a result solute-solute and solute-solvent interactions are markedly decreased. The present account discusses the nature and properties of cryptate cations; the available information indicates that for a suitable cryptandcation pair, the enclosed metal cation is effectively shielded from the environment. Cryptate formation allows the stabilization of unusual species (metal anions, unstable oxidation states, etc) and markedly influences chemical reactivity, either inducing anion activation or inhibiting cation participation. It represents a tool for studying ionic reactions in solution, while at the same time strongly affecting the usual course of reactions like acid-base equilibria, phase transfer catalysis, alkylations, additions to carbonyl groups, nucleophilic substitutions, carbanion reactions, anionic polymerizations, etc.
\end{abstract}

In condensed phase, liquid or solid, the interactions of a chemical species with its environment play a major role in determining its physical properties and its chemical reactivity. Marked changes may be brought about by introduction of a complexing agent, thus transforming a two-body interaction, solute-solute or solute-solvent, into a three-body interaction, substrate-ligand-solute or substrate-ligand-solvent. It is well known that such complexation greatly affects the properties of the bound species and that the resulting complex displays a wealth of new properties of its own, depending on the nature of the substrate-1igand pair considered.

Within this vast domain, the present discussion will bear on a specific combination:

- ligands forming inclusion complexes, cryptates, in which the substrate is bound in a cavity inside an organic molecule and therefore more or less effectively shielded from its environment in the free state;

- metal cation substrates, focusing mainly on alkali cations (AC's) which act as positive ions in many chemical phenomena involving ionic processes.

Strong and selective complexes of $\mathrm{AC}^{\prime} \mathrm{s}$ have only been obtained in rather recent times, with the discovery of new types of ligands. The most stable ones are found among the cryptates formed by inclusion of the cation into a macropolycyclic ligand (cryptand) (1-3). Efficient complexation is also displayed by the macrocyclic polyethers, "crown" compounds (4), and by macrocyclic substances of natural origin (5).

Within the first class of species, most studies have been performed to date with three macrobicyclic molecules, the cryptands I [2.1.1], II [2.2.1] and III [2.2.2] (6-7), which have been the most widely available. They contain a spheroildal cavity of size increasing 
from I to III, and form highly stable and selective cryptates by inclusion of spherical AC's, showing preference for $\mathrm{Li}^{+}, \mathrm{Na}^{+}$and $\mathrm{K}^{+}$respectively (8). Their physical and chemical properties have been studied in detail and will not be reviewed here (1-3, 6-8). Structure $\mathrm{V}$ shows a schematic representation of a cryptate $\left[\mathrm{M}^{\mathrm{n}+} \subset 2.2 .2\right]$.

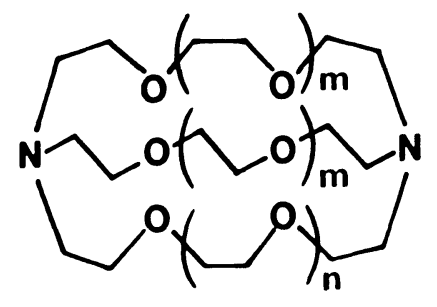

I [ 2.1.1] m=0, $\mathrm{n}=1$

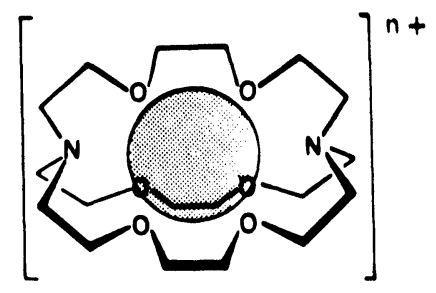

V

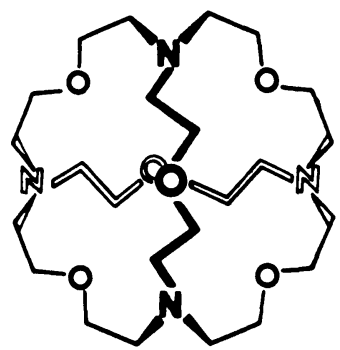

VI

II $[2.2 .1] \mathrm{m}=1, \mathrm{n}=0$

III [ 2.2.2] $\mathrm{m}=\mathrm{n}=1$

IV $\left[2.2 . \mathrm{C}_{8}\right] \mathrm{m}=1$,

0 replaced by $\mathrm{CH}_{2}$

in third bridge

The area is of course not limited to these ligands, nor to the complexation of AC's. The spherical macrotricycle VI contains a larger cavity than I-III, well suited for $\mathrm{Cs}^{+}$ complexation (9). Transition metal cryptates, macrotricyclic cryptand molecules forming dinuclear cryptates, as well as anion cryptates and complexes of organic substrates represent other facets of the chemistry of macropolycyclic inclusion complexes (see ref. 1,2 and references therein).

The studies on the physical and chemical properties of cryptates and of their effect on chemical reactivity cover various fields of chemistry: organic, physical, inorganic, biological chemistry. The present discussion is aimed at analyzing some of the results obtained in various laboratories, which bear mainly on the aspects of cryptate chemistry related to the problem of solute-solute and solute-solvent interactions of ionic type. Although brief and non- exhaustive, such an analysis should help bringing together investigations in different areas and outlining the contribution of cryptates to the major themes of research in the field.

\section{THE CRYPTATE CATION}

Cryptate formation amounts to inclusion of a cation into a molecular cavity, thus transforming a small inorganic cation into a very large almost spherical organic cation (Fig. 1). As a result, one may expect the included species to be more or less well s..2ielded from the other solutes ( $e . g$. the counter-anion) and from the solvent molecules, depending on the nature of cryptand-cation pair.

Crystal structure determinations $(10,11)$ and Stokes' radii from conductivity studies (12) indicate that the [2.2.2] cryptates are indeed very large cations of almost spherical shape and of about $5 \AA$ radius (for comparison the $\mathrm{NBu}_{4}{ }^{+}$cation has a radius of $4.1 \AA(12)$ ). Considering that the ionic radius changes only by about $0.9 \AA$ from $\mathrm{Li}^{+}(0.78 \AA)$ to $\mathrm{Cs}^{+}$ $(1.69 \AA)$, the change from $\mathrm{Cs}^{+}$to $\left[\mathrm{K}^{+} \subset 2.2 .2\right]$ is very large, about $3.5 \AA$ (Fig. 1 ). 

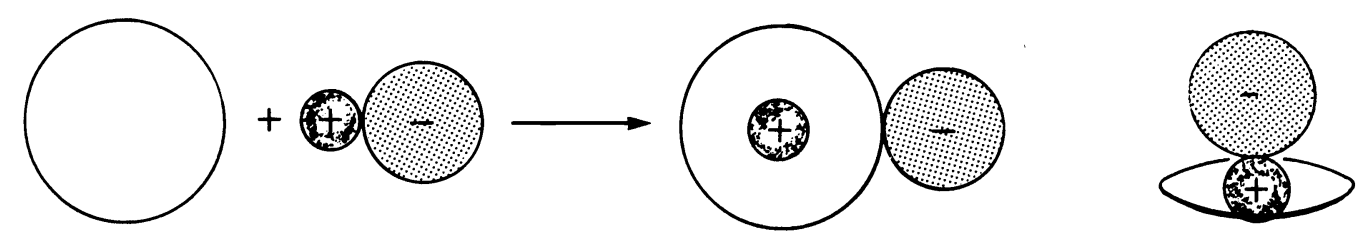

Fig. 1. Schematic representation of cryptate formation converting an alkali cation (here about $1.5 \AA$ radius) into a large spheroỉdal organic cation of about $5 \AA$ radius, with formation of an almost complete "organic skin" around the metal ion. At right: schematic representation of ion pairing in a macrocyclic complex.

Such a cryptate cation should have very low surface charge density and very weak interactions with either anions or solvent molecules. It may be considered as an extrapolation of $\mathrm{Cs}^{+}$to very large ionic size, with resulting strong exaltation of the weakening of cation-anion and cation-solvent interactions found along the series of alkali cations from $\mathrm{Li}^{+}$to $\mathrm{Cs}^{+}$. In other words, $\left[\mathrm{K}^{+} \subset 2.2 .2\right]$ and cryptate cations in general, might formally be considered as "super-heavy" alkali cations.

Along the series of $\mathrm{Na}^{+}, \mathrm{K}^{+}, \mathrm{Rb}^{+}$and $\mathrm{Cs}^{+}$cryptates of $[2.2 .2]$, the $\mathrm{M}^{+} \ldots 0$ distance changes by $0.4 \AA$ (from $2.57 \AA$ to $2.97 \AA$ ) (10), i.e. less than the change in radius of $\sim 0.9 \AA$ along the series of free cations. Furthermore, since the cryptates are much larger, their surface charge density varies much less than that of the AC's. Thus, in this respect they appear to be similar species, and the major differences among them should result from how well the complexed cation is still accessible to interactions with the environment.

A study of the volumes of complexation showed that formation of $\left[\mathrm{Li}^{+} \mathrm{C} 2.2 .2\right]$ leads to a contraction of the ligand while an expansion is found for the other AC cryptates of [2.2.2]. These changes in ligand volume increase linearly with cation size except for $\mathrm{Cs}^{+}$, a possible indication of incomplete inclusion of this large cation (13). Because of their shape and size, an interesting bonus of cryptate cations is their usefulness in crystal structure determinations of large complex anions, both because they appear to facilitate crystallization and to yield isolated anions (14).

\section{THE CRYPTATE CATION IN ITS ENVIRONMENT}

How well is the cryptated cation shielded from counterions and solvent molecules? An important factor in this respect is the polycyclic structure of cryptands. With macrocyclic crown ligands, the encircled cation is still accessible for ion pairing with an anion from "top" and "bottom" of the complex. This is much more difficult for the well encased cations in the cryptates (Fig. 1). Such a difference in environmental accessibility, which results from interposing a more or less complete "organic skin", may be expected to play a major role in all properties associated with the anion accompanying the complexed cation.

Ion pairing in the solid state is indeed found in the crystal structures of crown complexes (15). This is not the case for most alkali cryptates $(10,11)$, where the anions are usually far from the included cation. However, it should be noted that interaction through a face of the macrobicyclic ligand in a cryptate is sterically possible when the face in question is an eighteen-membered ring (like the [2.2] rings in [2.2.2] and [2.2.1]) or a larger one (like in [3.2.2] etc.). Indeed, the crystal structures of the doubly and triply charged [2.2.2] cryptates of $\mathrm{Ba}^{2+}, \mathrm{Pb}^{2+}(16), \mathrm{La}^{3+}, \mathrm{Eu}^{3+}(17-18)$ show that the metal cation completes its ligation shell by interacting with water molecules and/or anions through the faces of the macrobicycle. In the $\left\{[\mathrm{La} C 2.2 .2]\left(\mathrm{NO}_{3}\right)_{2}\right\}^{+}$species, the $\mathrm{La}^{3+}$ cation is 
coordinated to the eight heteroatoms of the cryptand as well as to two nitrate ions, bound in a bidentate fashion through two faces of the macrobicycle (17). A nice illustration of this point is provi $d$ by the [2.2.1] cryptates of NaSCN and KSCN. Whereas the crystal structure of $\left[\mathrm{Na}^{+} \subset 2.2 .1\right] \mathrm{SCN}^{-}$consists of separated cations (contained in the cavity) and anions, in the case of $\left[\mathrm{K}^{+} \subset 2.2 .1\right]$ the cation is too large and protuding through the [2.2] face of the cryptand thus allowing interaction with the $\mathrm{SCN}^{-}$anion (19).

of course, one would not expect total shielding of the cryptand cation, whatever the ligand and the cation. But there are numerous data obtained by a variety of methods, which indicate that cryptates provide the closest approach to an isolated metal cation. In the $\left[\mathrm{Li}^{+} \mathrm{C} 2.1 .1\right]$ cation, interaction with either solvent or anion is rendered very difficult by the small ring size of the faces. Indeed, this cryptate is separated from the anion in the solid state (20) and its ${ }^{7} \mathrm{Li}$ NMR signal is solvent independent, while the shifts of the free $\mathrm{Li}^{+}$resonance cover a range of $1.5 \mathrm{ppm}$ (21). In contrast, and not unexpectedly, the shifts of the $\mathrm{Li}^{+}$cryptates of [2.2.1] and [2.2.2] are definitely solvent dependent (21).
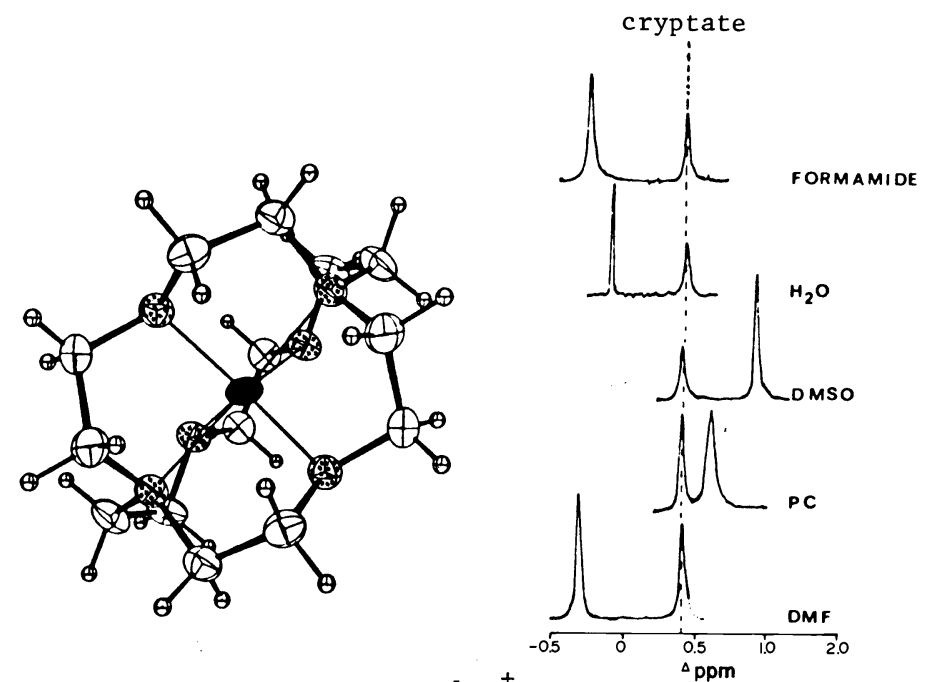

Fig. 2. (Left): Crystal structure of the $\left[\mathrm{Li}^{+} \mathrm{C} 2.1 .1\right]$ cryptate (20). (Right):

${ }^{7} \mathrm{Li} \mathrm{NMR}$ of free $\mathrm{Li}^{+}$cation and $\left[\mathrm{Li}^{+} \mathrm{C} 2.1 .1\right]$ cryptate in various solvents (21).

NMR of alkali cations has in general been a very useful tool for studying cation solvation and complexation (22). Thus, whereas the ${ }^{23} \mathrm{Na}$ resonance of the free cation is strongly solvent dependent that of $\left[\mathrm{Na}^{+} \mathrm{C} 2.2 .2\right]$ is only little affected (22). On the other hand, the recognition of a solvent dependent and of a solvent independent component in the ${ }^{133} \mathrm{Cs}$ NMR shift of $\mathrm{Cs}^{+}$in presence of [2.2.2] cryptand, led to the formulation of an equilibrium between an "inclusive" and an "exclusive" complex for this cation, which, being too large for the cavity of the ligand, must distort it on inclusion (23). A very sensitive test of the efficiency of a ligand to shield a cation is provided by thallium-205 NMR studies of $\mathrm{Tl}^{+}$complexes. The solvent dependence of the shifts of the free $\mathrm{Tl}^{+}$ion covers over $2600 \mathrm{ppm}$ (24). While the solvent change from DMSO to pyridine shifts the $\mathrm{TlCO}_{4} 1$ ine by $295 \mathrm{ppm},\left[\mathrm{Tl}^{+} \subset 2.2 .2\right]$ is shifted by on $1 \mathrm{y} 18 \mathrm{ppm}$ ! Furthermore, the resonance of the $\mathrm{Tl}^{+}$ complex of 18-crown-6 shows a strong solvent dependence indicating that the cation is able to interact markedly with both the solvent and the anion (25).

The far infrared spectra of sodium and lithium cryptates contain a broad band which arises from the vibration of the cation in the cryptand cavity; its frequency is independent of the solvent, whereas shifts of about $70 \mathrm{~cm}^{-1}$ are found for the free cations (26). Altering the normal mixture of ion sites of a solution by adding cryptands provides a useful 
tool for the interpretation of their Raman and IR spectra $(27,88)$. Conductance studies showed that interaction of $\left[\mathrm{K}^{+} \subset 2.2 .2\right]$ and $\left[\mathrm{Na}^{+} \mathrm{C} 2.2 .1\right]$ with the solvent is very weak (12).

The efficiency of cryptands for breaking ion pairs is clearly illustrated by much higher dissociation constant of terBuOK in terBuOH when $\mathrm{K}^{+}$is complexed by [2.2.2] as compared to dibenzo-18-crown-6 (28) and by the increase, by a factor of about $10^{4}$, of the dissociation constant of carbazyl sodium and potassium in THF on addition of [2.2.2] (29,30). Along the same lines, the absorption spectrum of difluorenylbarium in THF shows the presence of only contact ion pairs; addition of dimethyldibenzo-[18]-crown-6 gives a $1: 1$ mixture of contact and separated ion pairs, whereas only the latter are present when [2.2.2] is added (31). ESR studies of semidione radical anions showed that addition of the suitable cryptand, corresponding to the alkali cation involved, leads to complete dissociation of the chelated semidione-alkali cation ion pair $(32,33)$. In the case of the (cyclopenta-1,2-dione radical anion) $-\mathrm{K}^{+}$species, addition of [2.2.2] gives a ratio (free ion)/(ion pair) $>20$, while it is $<0.05$ when 18 -crown-6 is used (33).

Although cryptate formation very strongly increases the dissociation constants with respect to the uncomplexed salt, cryptated ion pairs are present in low polarity solvents, as shown by conductance measurements $(29,30)$. Thus, despite the large Stokes' radii of cryptate cations and of the resulting large interionic distance, sufficient interaction is still present for ion pairing to occur.

Thermodynamic studies indicate that the enthalpies, entropies and free energies of transfer of $\left[\mathrm{M}^{+} \mathrm{C} 2.2 .2\right]$ cryptates $\left(\mathrm{M}^{+}=\mathrm{Li}^{+}, \mathrm{Na}^{+}, \mathrm{K}^{+}, \mathrm{Rb}^{+}, \mathrm{Cs}^{+}, \mathrm{Ag}^{+}\right)$from water to methanol vary considerably with the central cation (34); constancy of the transfer parameters had been proposed earlier (35)., It appears that, although the metal cations are efficiently shielded, the alkali cryptates are still different enough with respect to thermodynamic parameters, to prevent general validity of single-ion assumptions. We have seen above from crystal structures and alkali ion NMR, that interactions with the medium may be present depending on the case considered. This is for instance especially true for the $\mathrm{Li}^{+}$and $\mathrm{Cs}^{+}$ cryptates of [2.2.2]. On the other hand, one may note that the enthalpies of transfer of the alkali cryptates of [2.2.2] (except for $\mathrm{Li}^{+}$) differ less from one another than those of the quaternary ammonium cations. In a number of instances, a comparison has been drawn between $\mathrm{R}_{4} \mathrm{~N}^{+}$and cryptate cations.

From the above discussion one may conclude, that cryptate formation results in an efficient shielding of the complexed cation from the environment, with a large decrease in interactions with anions and solvent molecules. However, it is also clear, that the efficiency of this cryptate effect depends on the specific cryptand-cation pair considered and that, although considerably weakened, some interaction does of course remain.

\section{PHYSICAL CHEMISTRY OF THE CRYPTATES}

Some physico-chemical properties of the cryptates have already been discussed in relation to the interactions with the medium. Many other features have been studied $(2,3)$ of which some are especially relevant to the present theme.

Cryptates represent a new approach to the study of ionic solvation. The coordination shell of cryptands surrounds the cation and is not subject to fast exchange, like solvent molecules usually are, thus providing cations in "frozen" solvation shells whose binding sites and geometry may be modified at will.

A double nuclear spin probe method using ${ }^{13} \mathrm{C}$ and ${ }^{23} \mathrm{Na}$ relaxation time measurements allowed the determination of ${ }^{23} \mathrm{Na}$ nuclear quadrupole coupling constants and electric field 
gradients in the different coordination shells of several cryptates (36), yielding a linear relation between ${ }^{23} \mathrm{Na}$ shift and quadrupole coupling constant $\chi$ :

$$
X=(0.05 \delta+1.65) \mathrm{MHz} \quad\left(\delta={ }^{23} \mathrm{Na} \text { shift from } \mathrm{NaCl}\right)
$$

This equation allows the determination of field gradients at the ${ }^{23} \mathrm{Na}$ nucleus in various solvents by simply measuring ${ }^{23} \mathrm{Na}$ shifts; the field gradient in turn leads to the mobility of the cation from the ${ }^{23} \mathrm{Na}$ relaxation time (37). Thus, a detailed study of ${ }^{23} \mathrm{Na}$ solvation becomes possible. The method should be extendable to other quadrupolar nuclei.

The stability constants of cryptates and the cation exchange kinetics also reflect the special nature of these ligands (see ref. 1-3 and references therein). A specially clear illustration of the cryptate effect on cation complexation is found in the high preference of the cryptand IV [2.2. $\mathrm{C}_{8}$ ] for $\mathrm{K}^{+}$versus $\mathrm{Ba}^{2+}$ (ratio $>10^{2}$ ) whereas [2.2.2] itself (which provides two more $\mathrm{O}$ binding sites) and the monocyclic ligand $\mathrm{N}, \mathrm{N}$-dimethyl-[18] $-\mathrm{N}_{2} \mathrm{O}_{4}$ (which allows approach of solvent to the bound cations) both complex $\mathrm{Ba}^{2+}$ much more strongly than $\mathrm{K}^{+}\left(\mathrm{Ba}^{2+} / \mathrm{K}^{+}>10^{2}\right)$. Such additional solvation is hindered in the complexes of $\left[2.2 . \mathrm{C}_{8}\right](38)$.

The redox potentials of the cations are strongly affected by cryptate formation. Electrochemical reduction of alkali, alkaline-earth and transition metal cryptates occurs at appreciably more negative potentials than those of the free cations, the difficulty of reduction increasing with the stability constant (39). A striking stabilization of the Eu II state (by $370 \mathrm{mV}$ ) with respect to $\mathrm{Eu}^{\text {III }}$ has been found for the europium cryptate of [2.2.2] (40). Thus, cryptates may stabilize unusual oxidation states and modify electron transfer rates. Further studies of transition metal cryptates (mononuclear or polynuclear) may be of great interest in this respect.

\section{CRYPTATE COUNTERIONS IN METAL SOLUTIONS. METAL ANIONS}

Since cryptates are very large cations (see above), if the associated anion is an electron, the resulting species is formally a very large "alkali" metal*. Rough extrapolation of ionization potentials from $\mathrm{Li}^{+}$to $\mathrm{Cs}^{+}$to $\left[\mathrm{K}^{+} \subset 2.2 .2\right]$ as a function of ionic radius shows that such a metal should have very low ionization potential $(<1 \mathrm{eV})$. It should therefore be very strongly reducing and contain an almost free electron. Electrochemical reduction of alkali and alkaline-earth cryptates gave evidence for the primary formation of unstable, short-lived intermediates like $\left[\mathrm{K}^{\circ}, 2.2 .2\right]$ which then dissociate (39).

Macrocyclic polyethers of the crown type and cryptands like [2.2.2] have had a strong impact on the field of metal solutions. Indeed, they strongly enhance the solubility of alkali metals (and even of barium in the case of [2.2.2]) in a variety of solvents (43). Due to the much higher stability of the cryptates, [2.2.2] is more efficient than the crown compounds. Whereas alkali metal solutions obtained with the latter mainly contain the metal anion $\mathrm{M}^{-}$, [2.2.2] yields in addition substantial concentrations of solvated electrons. Electronic absorption spectra taken at various stages of potassium metal dissolution by [2.2.2] in THF show the formation first of solvated electrons and thereafter, progressively as more metal dissolves, of $\mathrm{K}^{-}$by reduction of $\mathrm{K}$ by the solvated electrons; when the dissolution of the different alkali metals is compared,the largest amount of solvated electrons is obtained for the most stable cation-cryptand pair (Fig. 3) (44).

\footnotetext{
* For analogies, see the species $\mathrm{Li}\left(\mathrm{NH}_{3}\right)_{4}$ described as $\left\{\left[\mathrm{Li}^{+}\left(\mathrm{NH}_{3}\right)_{4}\right] \mathrm{e}^{-}\right\}(41)$, and $\mathrm{N}\left(\mathrm{CH}_{3}\right)_{4}$, formed by electrochemical reduction of the cation (42).
} 


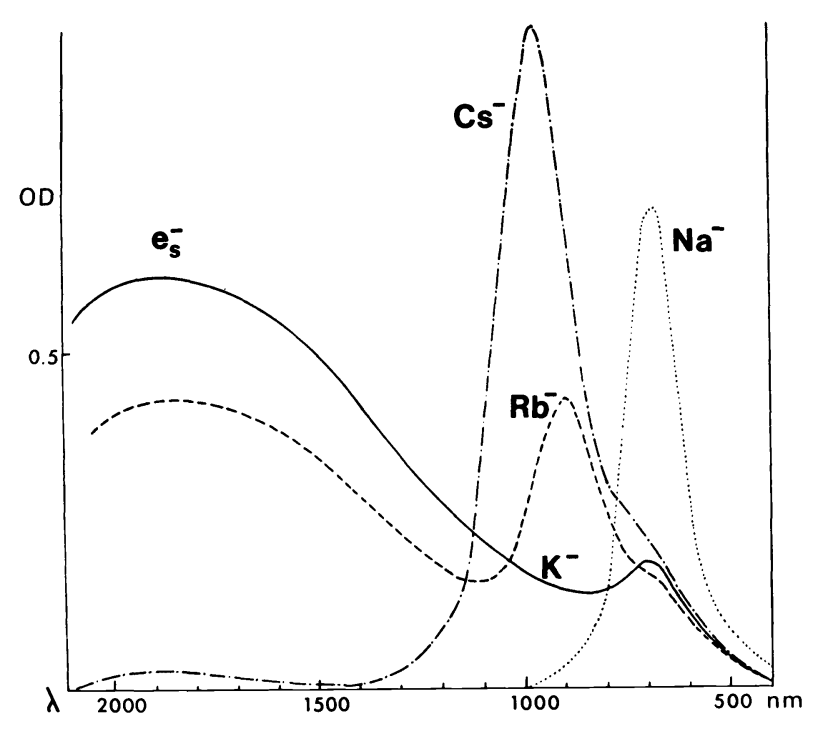

Fig. 3. Electronic spectra showing the formation of solvated electrons and alkali metal anions by dissolution of the corresponding metals in THF (at $-50^{\circ}$ ) using cryptand [2.2.2] (44). (Some $\mathrm{Na}^{-}$species in present in the spectra of the other alkali anions).

When the benzene and toluene radical anions were generated by dissolution of potassium in these solvents, the solutions obtained with dicyclohexyl-18-crown-6 displayed the usual proton hyperfine splitting of the radical anion, whereas a time-averaged ESR line was obtained with [2.2.2] $(44,45)$. This is due to slow electron exchange between the radical anion and solvent molecules in the crown case and fast exchange in the cryptate case. These results may be taken as evidence for ion pairing between the radical anion and the accessible cation complexed by the crown ligand (46), whereas such pairing is much weaker with [2.2.2] resulting in a much less stable, rapidly exchanging, radical anion.

The most striking illustration of the reducing power of cryptate metal solutions and of cryptate cation ability to stabilize unusual species is the isolation by Dye et al. of the first salt containing an alkali metal anion $\left[\mathrm{Na}^{+} \mathrm{C} 2.2 .2\right] \mathrm{Na}^{-}$as crystals of gold colored shiny metallic appearance $(43,47,48)$. Its crystal structure has been determined and its ${ }^{23} \mathrm{Na}$ NMR spectrum contains both a broad $\mathrm{Na}^{+}$resonance at the expected position and a narrow, upfield $\mathrm{Na}^{-}$resonance. Potassium, rubidium and cesium yield gold-colored solids and films with [2.2.2] which contain the corresponding metal anions. When stoichiometric quantities of [2.2.2] and metal are used, strongly paramagnetic blue films are obtained, which may be formulated as the electride $\left[\mathrm{M}^{+} \subset 2.2 .2\right] \mathrm{e}^{-}(43,48,49)$. The characterization of a new oxidation state of the alkali metals is certainly not limited to the sole [2.2.2] cryptand. Solid ceside salts for instances may be accessible with larger cryptands like [3.2.2] or macrotricyclic ones which accomodate $\mathrm{Cs}^{+}$.

Other new types of metal ions may also become isolable using cryptate counterions, a particularly attractive class of substances being that of anionic clusters, both because of their fundamental interest and of their potential catalytic properties. Crystalline salts containing polyatomic anions of the heavy post-transition metals $\left(\mathrm{Sb}_{7}{ }^{3-}, \mathrm{Bi}_{4}{ }^{2-}, \mathrm{Ge}_{9}{ }^{2-}\right.$, $\mathrm{Ge}_{9}{ }^{4-}, \mathrm{Pb}_{5}{ }^{2-}$ and $\mathrm{Sn}_{9}{ }^{4-}$ ) with $\left[\mathrm{Na}^{+} \mathrm{C} 2.2 .2\right]$ as counterions have been obtained and their crystal structure has been determined (50); in these species complexation of $\mathrm{Na}^{+}$by [2.2.2] prevents reversion to the starting $\mathrm{Na} / \mathrm{metal}$ alloy phase. 
These results show that [2.2.2] and presumably other cryptands, provide access to new chemistry of metals and electrons in solution and in the solid state. They also agree with formally viewing the "electride" type cryptates $\left[\mathrm{M}^{+} \subset 2.2 .2\right] \mathrm{e}^{-}$as very large, spherical, "expanded-metal" type entities of very high reducing power (see above)* . The chemical properties of the radical anions, metal anions and metal solutions obtained with cryptands, their use in organic and inorganic chemistry, desserve further investigation.

\section{CRYPTATES AND CHEMICAL REACTIVITY}

One of the initial motivations of our work on cryptates (51) was the expectation that cation complexation by a cryptand should bring about solubilization of the salt in solvents of low polarity in which it would be otherwise insoluble, and dissociation or separation of the cation-anion pair. As a result, ionic reactions may be affected in two opposite ways: anion activation may be brought about; cation participation may be inhibited (or at least strong1y hindered).

\section{ANION ACTIVATION BY CRYPTATE FORMATION}

Cryptate formation transforms an alkali cation into a voluminous organic cation which is much more soluble in organic medium. The anion accompanying the cryptate will be activated since both ion pairing with the large cation and solvation by organic molecules of low polarity provide only little stabilization. Thus, almost "naked" anions, i.e. anions interacting only weakly with the environment, may be obtained. Anion activation is potentially of great interest for energy conservation and for decreasing thermal pollution. Such is the case with any reaction which, under activation, becomes feasible at room temperature while usually requiring heat, especially if the activation is catalytic. The extensive work on the use of crown type macrocyclic polyethers in organic reactions will not be discussed, but only mentioned for comparison purposes when appropriate.

Cryptate formation may lead to a large increase in rate of anionic reactions for two reasons: activation causes an increase in reactivity of the reagent, i.e. an increase in rate constant, while solubilization increases its concentration. However, activation and solubilization have opposite requirements. High anion activation requires large cation-anion separation and little anion solvation, but then solubility in non-polar media is expected to decrease as activation increases. In order 1) to impose large cation-anion separation (i.e. weak ion pairing), while 2) retaining high solubility in media of low polarity, ligands are

\footnotetext{
* Such species are of interest to the physics of the metallic state since they may yield information on the behaviour of the metallic properties as the size of the cation is strongly increased. The "electrides" obtained with [2.2.2] $(43,48,49)$ contain a very large cation (about $5 \AA$ radius) and are paramagnetic solids. They could be considered as containing electrostatically trapped electrons (see also $\mathrm{NR}_{4}$ species (42)). On the other hand, $\mathrm{Li}\left(\mathrm{NH}_{3}\right)_{4}$ which contains the smaller complex cation $\left[\mathrm{Li}\left(\mathrm{NH}_{3}\right)_{4}\right]^{+}$(radius of about $3.7 \AA$ ) has metallic properties (41). Does this mean that there is a transition from metallic to paramagnetic state somewhere between cation radii of 3.5 and $5 \AA$ ? Of course the chemical nature of the ligand surrounding the cation plays a role in addition to mere size. For instance, the electride of [ $\mathrm{Li} \subset 2.1 .1]^{+}$, which has a smaller radius than [2.2.2] cryptates, would be worth investigating. Also, it might become possible to devise species located at the frontier between metallic and paramagnetic states. One might mention the analogy, in terms of size and formal radius of the orbit of the associated electron, between the electrides and Rydberg atoms (R.F. Stebbings, Science 193, 537-542 (1976)). Other directions of research related to the chemistry of metals and electrons are being explored : - cryptands providing both a cation complexing site and an electron acceptor site should be able to form expanded metal type molecular species; - "electron cryptates" might be realizable by trapping electrons within a suitably designed molecular structure.
} 
required which 1) are very thick and wrap entirely around the cation, 2) have high liposolubility. Fortunately, thickness and liposolubility are compatible features and may be achieved for instance by attaching hydrocarbon chains to [2.2.2] as in compounds VII and VIII, which have much improved salt solubilization properties (52-54). The bulky and 1ipophilic cryptand IX containing three fused cyclohexyl rings, appears especially well suited in these respects (55).

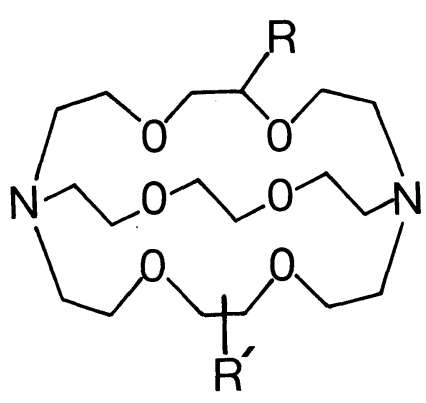

VII $\mathrm{R}=-\left(\mathrm{CH}_{2}\right) n^{-\mathrm{CH}_{3} ; \mathrm{R}^{\prime}=\mathrm{H}}$ VIII $\mathrm{R}=\mathrm{R}^{\prime}=-\left(\mathrm{CH}_{2}\right){ }_{n}-\mathrm{CH}_{3}$ $(n=9,10,13,19)$

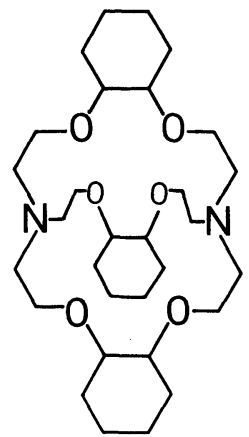

IX

As discussed above, ion pairing with direct contact of the ions is still possible between crown complexed cations and anions, while such direct pairing is weaker or absent when [2.2.2] or other cryptands are used in the same systems. Thus, strictly speaking, "naked" anions are not formed with the macrocycles (but complexed ion pairs) or at least to an appreciably lesser extent than with the macrobicycles. In fact, even with cryptate cations, ion pairing still occurs, forming cryptated ion pairs as shown by conductivity measurements (see above) $(29,30)$. This simply means that as long as the anion is not isolated in the gas phase, it will interact with whichever species is present, however small this interaction may well be. The larger the anion-cation separation imposed by the cryptand and the weaker the interactions with the solvent, the closer one may come to performing gas phase type chemistry in solution.

With highly localized anions, solubilization may be very difficult. Thus, stoichiometric dissolution of salts containing such hard anions as fluoride or hydroxide in benzene cannot be achieved even by lipophilic [2.2.2] derivatives of type VII, unless a molecule of $\mathrm{CHCl}_{3}$ is present for solvating $\mathrm{F}^{-}$(53). The same holds for crown macrocycles (56).

Strongly basic systems (57) are obtained by addition of [2.2.2] to solutions of sodium tertioamylate in benzene or of butyl-1ithium in hexane. Neither solution ionizes tripheny1methane or diphenylmethane, whereas anion formation is immediate on addition of [2.2.2] That dissociation of the strongly associated ions has occured is also shown by a very 1 arge increase in conductivity of the tertioamylate solution. A study of the $\mathrm{pK}_{\mathrm{a}}$ of the ter-Am0 anion with the potassium cation complexed by a cryptand VII in the presence of p-pheny1toluene as "indicator" gave an approximate value of $>37$ (53). Cryptands may be used for determining very low acidities (58) and cryptate-enolate solutions are strong bases (59,60). Activation of alkali metal hydrides may occur (60). It would be very interesting to be able to produce a cryptate hydride like $\left[\mathrm{M}^{+} \subset 2.2 .2\right] \mathrm{H}^{-}$; however such a species is expected to be very reactive, and unstable, since it would contain an almost free hydride ion. Some preliminary experiments indicated destruction of the ligand on attempted dissolution of $\mathrm{KH}$ with [2.2.2] (61), but suitable conditions might well be found. 
The high1y hindered ester, methyl mesitoate, is hydrolyzed ( $70 \%$ yield, $12 \mathrm{~h}$ at $25^{\circ}$ ) in the presence of [2.2.2] by powdered $\mathrm{KOH}$ suspended in dry benzene (57), a reaction much faster than that reported in the presence of a crown ether $\left(58 \% ; 31\right.$ hours at $\left.73.8^{\circ}\right)(62)$; the reaction is even much more rapid when the concentrated solution of KOH in DMSO obtained with [2.2.2] is employed (57). In presence of cryptands, complete addition of the anion of alkali salts to pentacoordinate phosphoranes occurs, producing hexacoordinate phosphates (63).

Because of their solubilization and activation properties, cryptands are of use in phase transfer catalysis (PTC) either from solid to liquid or from liquid to liquid. Catalytic amounts of [2.2.2] bring about high yield substitution reactions, like ester formation from potassium carboxylates $(64,65)$, fluorination (66), thiocyanation (57), alkylation of phenolates (67) and alcoholates (68).

Lipophilic cryptands VII-VIII were found to be the most efficient reagents for liquidto-1iquid (water-to-organic solvent) PTC in a variety of reactions (substitutions, reductions, cyclopropanations) $(52,54)$. Detailed comparative studies of anion reactivity and nucleophilicity indicate that lipophilic cryptates produce large reaction rate enhancements and a reactivity scale of the anions identical with the sequence in dipolar aprotic solvents. It was concluded that they probably represent the best approach to a model of solvent-separated ion pairs and that the reactivity shown by the anions should approach the maximum obtainable in solution (54). The bulky cryptand IX is somewhat more active than ligands of type VII (55). The catalytic activity of the cryptand [2.2.2] is retained when immobilized polymer support, thus allowing easy recycling of the catalyst (69).

The rate and regioselectivity of alkylation of enolate (70) and phenolate anions (67) are strongly affected by cryptation. Addition of 18-crown-6 to the potassium enolate of ethylacetylacetate yields a complexed ion pair in which the anion has a " $U$ " shape and the cation is externally complexed. On the contrary, with [2.2.2] separated ions are obtained, the anion having " $\mathrm{W}$ " or "S-trans" shape. Alkylation of this enolate with ethyliodide, gives, with respect to the reaction in pure THF (where only $\mathrm{C}$ alkylation occurs), a rate increase by a factor of 100 and $3 \% 0$ alkylation in presence of $18-c r o w n-6$, and a much larger rate increase (by a factor of 4000) and $21 \% 0$ alkylation when [2.2.2] is used (70).

\section{INHIBITION OF CATION PARTICIPATION}

Anion activation results in increased rates of reaction. On the contrary, if a cation assists a reaction, its complexation will hinder its participation and is therefore expected to decrease reaction rates. Thus, cryptation should allow to ascertain the participation of cations in ionic reactions.

Such effects have been found in nucleophilic addition to the carbonyl group. The rate of reduction of carbonyl and other functional groups by $\mathrm{LiAlH}_{4}$ and $\mathrm{NaBH}_{4}$ is markedly slowed down or inhibited by cryptation of $\mathrm{Li}^{+}$or $\mathrm{Na}^{+}$with [2.1.1] or [2.2.1] respectively, showing that coordination of the cation to the carbonyl group and to the developping alkoxide plays a major role in the reaction $(71,72)$. Similarly, the addition of an organolithium compound to carbonyl groups is assisted by the $\mathrm{Li}^{+}$cation (73). The stereoselectivity of reductions is also affected by cation complexation (74).

Addition of a suitable cryptand results in the inversion of the regioselectivity of reduction of $\alpha, \beta$-unsaturated carbonyl groups by $\mathrm{LiAlH}_{4}$ or $\mathrm{NaBH}_{4}(75,76)$. Indeed, when conjugated cyclohexenone is treated with $\mathrm{LiAlH}_{4}$ in ether, the reduction is very fast and yields 98\% cyclohexeno1 (1,2-addition) and only $2 \% 1,4$-addition, whereas in the presence of 
[2.1.1] in the same conditions, it is much slower and gives predominantly 1,4 addition (76\%). Using 12-crown-4 as additive leads to much weaker effects. These results indicate that cation participation plays an important role in the rate and regioselectivity of reduction of $\alpha, \beta$-unsaturated carbonyl compounds; removal of the cation by cryptation favours formation of the less localized anion, the enolate, by hydride addition at $C-(\beta)$, whereas free $\mathrm{Li}^{+}$ favours addition at the carbonyl carbon by ion pairing with the developping localized alkoxide anion. Stereochemical effects of cryptands in Michael condensation have also been reported (77).

\section{THE ANION ACTIVATION/CATION PARTICIPATION BALANCE}

A striking illustration of the cation participation/anion activation balance in the effect of cryptation on ionic reactions, is provided by the addition of acetonitrile anion to benzaldehyde :

$$
\mathrm{PhCHO}+\mathrm{M}^{+}\left(\mathrm{CH}_{2} \mathrm{CN}\right)^{-} \longrightarrow \quad \mathrm{Ph}^{-} \mathrm{CHO}^{-}-\mathrm{CH}_{2} \mathrm{CN}, \mathrm{Li}^{+} \quad\left(\mathrm{THF},-80^{\circ}\right)
$$

When $\mathrm{M}^{+}=\mathrm{Li}^{+}$, addition of [2.1.1] markedly decreases the reaction rate, indicating that the dominant factor is cation participation. With $\mathrm{M}^{+}=\mathrm{K}^{+},[2.2 .2]$ addition increases the rate by predominant anion activation. Finally for $\mathrm{M}^{+}=\mathrm{Na}^{+},[2.2 .1]$ does not much affect the rate indicating an intermediate case where the two effects apparently cancel out (78).

Assistance by alkali cations has been detected in a similar way in the ring opening of ethylene oxide by fluorenylsodium type reagents, since addition of [2.2.2] decreased the rate of cleavage by factors of about $10^{2}-10^{3}(29,79)$. In contrast, the rate of cleavage of ethylene oxide by carbazyl potassium increases on addition of [2.2.2] (29), showing that in this case anion activation occurs by separating the cation from the carbazyl anion in which the charge is much more localized than in fluorenyl anion.

The general tenure of these results is that cryptate formation represents a powerful tool for analyzing the mechanism of ionic reactions involving complexable metal cations. Two limiting classes of reactions may be considered:

1) anion activation dominant reactions, for which cryptation of the cation increases the reaction rate by ion pair separation;

2) cation participation dominant reactions, for which cryptation of the cation decreases the rate, indicating that the cation assists the reaction by complexation with the substrate and/or stabilization of the transition state via electrostatic interaction with the charge developping on the substrate (electrostatic catalysis).

The balance between 1) and 2) is determined by the nature of the reagent anion and the product anion, assuming that the transition state occurs at about the same position along the reaction path. The situation is schematically represented in Fig. 4.

Case 1) obtains when the cation interacts more strongly with the reagent than with the product anion, for instance when the reacting anion is sufficiently localized for strong ion pairing; case 2) occurs when the reagent anion is delocalized and the product anion is localized so that ion pairing is weaker with the former than with the latter (cf. fluorenyl and alkoxide anions in the case of ethylene oxide cleavage $(29,79)$ ). In the case of reaction (A) above, the smal1 $\mathrm{Li}^{+}$cation interacts more strongly with the product alkoxide anion than with the starting delocalized $\left(\mathrm{CH}_{2} \mathrm{CN}\right)^{-}$species.

of course, this is a rather simplified dichotomic view of the situation, but it accounts for the general behaviour observed. A more detailed analysis, involving the determination of the reactivities of all species which may be present, should shed more light on the 

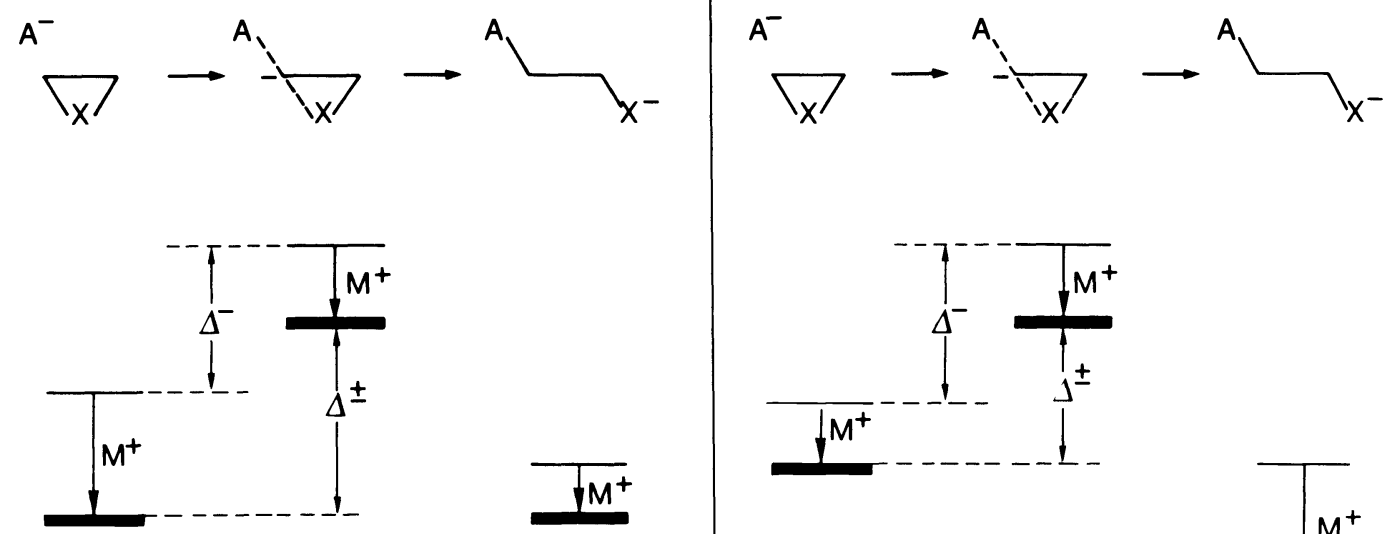

Effect of

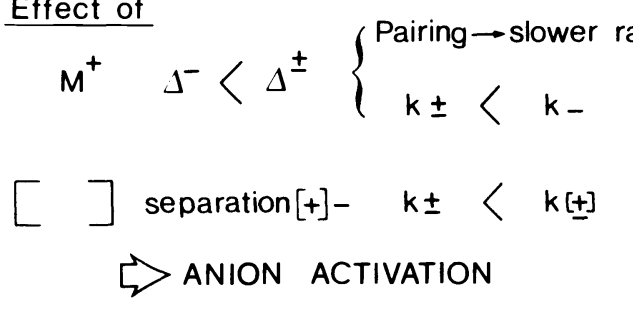

rate

Effect of

$\mathrm{M}^{+} s^{ \pm}<s^{-}\left\{\begin{array}{c}\text { Pairing } \rightarrow \text { faster rate } \\ \mathrm{k}_{-}<\mathrm{k}_{ \pm}\end{array}\right.$

[] separation $[+]-k \underline{t}<k \pm$ $\Leftrightarrow$ CATION ASSISTANCE

Fig. 4. Schematic illustration of the effects of ion pairing and ion pair separation by a cryptand [ ] on the reactivity of the free anion $A^{-}\left(k_{-}, \Delta^{-}\right)$, the ion pair $A^{-} M^{+}$ $\left(k_{ \pm}, \Delta^{ \pm}\right)$and the cryptate separated species $A^{-}\left[M^{+}\right]\left(k_{[ \pm]}\right)$. The opening of a threemembered heterocyclic ring by anion $\mathrm{A}^{-}$is taken as an example. The effect of pairing with metal cation $\mathrm{M}^{+}$on the energy of the initial state, the transition state and the product state is indicated by the vertical arrows for two idealized limiting cases.

(Left) Anion activation: stronger interaction of $\mathrm{M}^{+}$with a harder, more localized $\mathrm{A}^{-}$species than with the product; pairing decreases the reaction rate and separation by [ ] increases it.

(Right) Cation assistance: $\mathrm{M}^{+}$interacts more strongly with the product anion than with the softer, more delocalized or polarizable $\mathrm{A}^{-}$; pairing with free $\mathrm{M}^{+}$increases the reaction rate and cryptation by [ ] decreases it by inhibiting cation participation. It is assumed that the transition state is about half-way between initial and final states in both cases. 
intimate course of these ionic processes. For instance, separate determination of the reactivities of free anions, ion pairs and cryptated ion pairs indicated that the latter are less or more reactive than free ions depending on the nature of the nucleophile and of the substrate with which it reacts $(29,30,80)$. The higher reactivity of cryptated ion pairs may arise from assistance by the large cryptate cation when a large polarizable anionic species is involved. In the propagation reaction of propylene sulfide polymerization, the reactivity of the ion pair $\sim \mathrm{s}^{-}{ }^{+}$increases linearly with counterion size and becomes higher than the reactivity of the free anion when $\mathrm{M}^{+}$is a cryptate cation $(29,30)$.

Carbanion reactions form a class of reactions where the nature of the cation and its interaction with the anion play a primordial role. Cryptands may be expected to cause major effects and provide new insight into the behaviour of the various species present, for instance in reactions involving organometallic compounds. Thus, addition of [2.2.2] to butyllithium in hexane strongly activates the anion by breaking the $\left(\mathrm{Li}^{+} \mathrm{Bu}^{-}\right)$oligomeric associations (57). The $\alpha / \gamma$ regioselectivity of condensation reactions of the allylic carbanion $\mathrm{PhS}-\mathrm{CHLi}-\mathrm{CH}=\mathrm{CMe}_{2}$ with various substrates may change from mostly $\alpha$ to predominant $1 \mathrm{y}$ $\gamma$ on addition of [2.2.2] (81).

Carbanion rearrangements may be strongly affected. Thus, $\mathrm{PhCH}_{2}-\mathrm{S}-\mathrm{CHLiPh}$ may rearrange by two competing paths of either Stevens or Sommelet type. Whereas it undergoes exclusively Stevens type rearrangement in hexane or $\mathrm{THF}$, complexation of $\mathrm{Li}^{+}$by [2.2.2] leads to predominant Sommelet type rearrangement. This result may be explained'by noting that complexation should favour the pathway involving the more delocalized transition state, i.e. the Sommelet rearrangement (82).

Complexation experiments have also demonstrated the importance of internal chelation in the stereochemical properties of carbanions $\alpha$ to sulfoxide $(83,84)$. Many experiments may be imagined, lithium organic compounds being particularly important class of synthetic reagents. Use of the $\mathrm{Li}^{+}$specific [2.1.1] cryptand is attractive, since in the complex the $\mathrm{Li}^{+}$cation is especially well protected from external interactions (see above).

Finally, another reaction may be mentionned. The decarboxylation of potassium 6-nitrobenzisoxazole-3-carboxylate in benzene is strongly accelerated by addition of crown ethers and even more so by cryptand [2.2.2] (85). It was concluded that the macrocycle is externally complexed to the tight potassium carboxylate ion pair, while with the cryptate the ion pairing is considerably looser. Similar conclusions may be drawn from the electronic spectra of picrate salts in dioxane: the spectra of the crown complexed species are not very different from that of the tight ion pair, whereas the cryptate of potassium picrate has an absorption close to that of the loose ion pair of this salt.

A general conclusion pertaining to the effect of cation cryptation on ionic reactions in organic media is that it tends to favour the species or the process involving the most highly intermally stabilized anion (e.g. the most delocalized and/or polarizable anion), since external stabilization by ion pairing is reduced to a minimum.

\section{ANIONI.C POLYMERIZATION}

Anionic polymerization is an ideal field of application for cryptate induced anion activation, since new processes may be achieved while requiring only catalytic quantities of cryptand. Anion activation by cryptates allows the use of initiators which otherwise are unreactive and increases considerably the reactivity of living centres. Studies on the reactivity of the different species (free anions, ion pairs, cryptated ion pairs) present 
both in the initiation and in the propagation steps have been performed. They represent the most detailed analyses of the factors affecting the behaviour of these species and of the modifications brought about by cryptation. The polymerization of a number of monomers (isoprene, styrene, methyl-methacrylate, butadiene, ethylene oxide, propylene sulfide, isobutylene sulfide, 2-vinylpyridine, $\beta$-propiolactone, $\varepsilon$-caprolactone) has been effected using alkali metals or salts of radical anions and other anions in the presence of cryptands $(29,30,44,80,86)$. As initiators, alkali metal solutions containing alkali metal anions and solvated electrons were particularly interesting $(44,45,86)$. Some unusual reactions have been shown to occur, like nucleophilic additions of alkoxide anions on carbon-carbon double bonds. Addition of [2.2.2] to a solution of sodium tertioamylate and styrene in benzene causes formation of polystyrene, whereas no polymerization is observed in the absence of cryptand. This process must begin by a nucleophilic addition of the tertioamylate anion to styrene giving a benzyl anion. Another interesting example is the polymerization of hexamethyltrisiloxane by solid $\mathrm{KOH}$ in presence of [2.2.2], which induces a fast reaction yielding high molecular weight polysiloxanes $(86,87)$.

\section{CONCLUSION}

The effects of crown and cryptand type complexing agents on solute-solute and solutesolvent interactions have received wide attention from many laboratories and have broad applications in various areas of chemistry. The present account has stressed the contributions of the specific properties of cryptands and cryptates, both to physico-chemical studies of ionic species and to their chemical reactivity. Cryptates represent the closest approach yet, to very large complex cations of spherical type, as well as to isolated counteranions in the condensed phase. As such, cryptation exalts the modifications of physical and chemical properties encountered on solvation, chelation and complexation by crown macrocycles. The comparatively little studied spherical cryptates of the macrotricyclic ligand VI represent as for now the foremost step along the line. Many other fundamental investigations and practical uses may be imagined, either by employing presently available cryptands in new processes, or by tailoring new cryptands for a specific goal. For instance, the properties of di- or polynuclear cryptates and those of anion cryptates (2) represent promising areas of research. It is hoped that the present account on the work already performed and on the numerous interesting results obtained, will stimulate further studies, bringing new effects to light as well as providing new opportunities for applications.

\section{REFERENCES}

(1) J.M. Lehn, Structure and Bonding 16, 1-69 (1973).

(2) J.M. Lehn, Accounts Chem. Res. 11, 49-57 (1978); Pure Applied Chem. 49, 857-870 (1977), 50, 871-892 (1978).

(3) A.I. Popov and J.M. Lehn, in "Coordination Chemistry of Macrocyclic Compounds", G.A. Melson, ed., Chapter 9, P1enum Press New York (1979).

(4) C.J. Pedersen and H.K. Frensdorff, Angew. Chem. 84, 16-26 (1972); Angew. Chem. Intemat. Edit. 11, 16-25 (1972).

(5) Yu. A. Ovchinnikov, V.T. Ivanov and A.M. Shkrob, "Membrane Active Complexones", E1sevier, Amsterdam (1974).

(6) B. Dietrich, J.M. Lehn, J.P. Sauvage and J. Blanzat, Tetrahedron 29, 1629-1645 (1973).

(7) B. Dietrich, J.M. Lehn and J.P. Sauvage, Tetrahedron 29, 1647-1658 (1973).

(8) J.M. Lehn and J.P. Sauvage, J. Amer. Chem. Soc. 97, 6700-6707 (1975). 
(9) E. Graf and J.M. Lehn, J. Amer. Chem. Soc. 97, 5022-5024 (1975).

(10) B. Metz, D. Moras and R. Weiss, Chem. Comm. 444-445 (1971).

(11) D. Moras, B. Metz and R. Weiss, Acta Cryst. B29, 383-388, 388-395 (1973); D. Moras and R. Weiss, Acta Cryst. B29, 396-399 (1973).

(12) S. Boileau, P. Hemery and J.C. Justice, J. Solution Chem. 4, 873-891 (1975).

(13) N. More1-Desrosiers and J.P. Morel, Nouv. J. Chim. $\underline{3}$, 539-543 (1979).

(14) R.G. Teller, R.G. Finke, J.P. Collman, H.B. Chin and R. Bau, J. Amer. Chem. Soc. 99, 1104-1111 (1977); J.P. Collman, R.G. Finke, J.N. Cawse and J.I. Brauman, J. Amer. Chem. Soc. 99, 2515-2526 (1977); R.E. Ginsburg, R.K. Rothrock, R.G. Finke, J.P. Collman and L.F. Dah1, J.Amer.Chem.Soc. 101, 6550-6562 (1979); C. Caron, A. Mitschler, G. Rivière, L. Ricard, M. Schappacher and R. Weiss, J.Amer.Chem.Soc. 101, 7401 (1979).

(15) M.R. Truter, Structure and Bonding 16, 71-111 (1973).

(16) B. Metz, D. Moras and R. Weiss, J. Amer. Chem. Soc. 93, 1806-1808 (1971); B. Metz and R. Weiss, Inorg. Chem. 13, 2094-2098 (1974).

(17) F.A. Hart, M.B. Hursthouse, K.M. Abdul Malik and S. Moorhouse, J.C.S. Chem. Comm. 549-550 (1978).

(18) M. Ciampolini, P. Dapporto and N. Nardi, J.C.S. Chem. Comm. 788-789 (1978); J.C.S. Dalton 974-977 (1979).

(19) F. Mathieu, B. Metz, D. Moras and R. Weiss, J. Amer. Chem. Soc. 100, 4412-4416 (1978).

(20) D. Moras and R. Weiss, Acta Cryst. B29, 400-403 (1973).

(21) Y.M. Cahen, J.L. Dye and A.I. Popov, J. Phys. Chem. 79, 1289-1291 (1975).

(22) A.I. Popov, Pure Applied Chem. 51, 101-110 (1979); J.M. Ceraso, P.B. Smith, J.S. Landers and J.L. Dye, J: Phys. Chem. 81, 760-766 (1977).

(23) E. Mei, A.I. Popov and J.L. Dye, J. Amer. Chem. Soc. 99, 6532-6536 (1977);

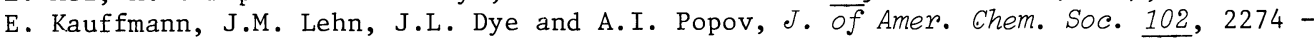
2278 (1980).

(24) J.J. Dechter and J.I. Zink, J. Amer. Chem. Soc. 97, 2937-2942 (1975).

(25) C. Srivanavit, J.I. Zink and J.J. Dechter, J. Amer. Chem. Soc. 99, 5876-5881 (1977).

(26) Y.M. Cahen and A.I. Popov, J. Solution Chem. $\underline{4}, 599$ (1975).

(27) P. Gans, J.B. Gill and J.N. Towning, J.C.S. Dalton 2202-2204 (1977).

(28) I.M. Kolthoff and M.K. Chantooni, Jr., Anal. Chem. 51, 1301-1306 (1979).

(29) P. Sigwalt and S. Boileau, J. Polymer Science: Polymer Symposium 62, 51-64 (1978).

(30) P. Hemery, W. Warzelhan and S. Boileau, Polymer 21, 77-80 (1980).

(31) J. Smid, Angew. Chem. Internat. Edit. 11, 112-127 (1972).

(32) G.A. Russe11, G. Wallraff and J.L. Gerlok, J. Phys. Chem. 82, 1161-1168 (1978).

(33) G.A. Russe11 and C.E. Osuch, J. Amer. Chem. Soc. 100, 5979-5981 (1978).

(34) M.H. Abraham, E.C. Viguria, A.F. Danil de Namor and T. Hill, Inorg. Chem. 19, 54-56 (1980); M.H. Abraham, A.F. Danil de Namor and R.A. Schultz,J.C.S. Faraday I 76, 869884 (1980); M.H. Abraham, A.F. Danil de Namor, H.C. Ling and R.A. Schulz, Tetrahedron Letters 961-964 (1980).

(35) S. Villermaux and J.J. Delpuech, J.C.S. Chem. Comm. 478-479 (1975); M.F. Lejaille, M.-M. Livertoux, G. Guidon and J. Bessière, Bull. Soc. Chim. France I, 373-377 (1978); J. Gutknecht, H. Schneider and J. Stroka, Inorg. Chem. 17, 3326-3329 (1978).

(36) J.P. Kintzinger and J.M. Lehn, J.Amer. Chem. Soc. 96, 3313 (1974).

(37) C. Detellier and P. Laszlo, Buzl. Soc. Chim. Belg. 84, 1087- (1975).

(38) B. Dietrich, J.M. Lehn and J.P. Sauvage, J.C.S. Chem. Comm. 15-16 (1973).

(39) J.P. Gisselbrecht, F. Peter and M. Gross, J. Electroanal. Chem. 74, 315-327 (1976); 96, 81-86 (1979); F. Peter, J.P. Gisselbrecht and M. Gross, ibid. 86, 115-140 (1978).

(40) O.A. Gansow, A.R. Kausar, K.M. Triplett, M.J. Weaver and E.L. Vee, J. Amer. Chem. Soc. 99, 7087-7089 (1977); E.L. Yee, O.A. Gansow and M.J. Weaver, ibid. 102, 2278-2285(1980).

(41) N. Mammano and M.J. Sienko, J. Amer. Chem. Soc. 90, 6322-6324 (1968).

(42) R.K. Quinn and J.J. Lagowski, J. Phys. Chem. 72, 1374-1375 (1968); J.D. Littlehailes and B.J. Woodha11, Discussion Faraday Soc. 45, 187-192 (1968).

(43) J.L. Dye in "Electrons and Fluids", J. Jortner and N.R. Kestner, Ed., p 76-95, Springer, Berlin (1973); J.L. Dye, Angew. Chem. 91, 613-615 (1979); Angew. Chem. Internat. Edit. 18, 587-598 (1979); J.L. Dye, in "Progress in Macrocyclic Chemistry", 
vo1. 1, R.M. Izatt and J.J. Christensen, Ed., p 63-113 (1979).

(44) M. Viguier, M. Abadie, F. Schué and B. Kaempf, European Polymer J. 13, 213-222 (1977); J. Lacoste, F. Schué, S. Bywater and B. Kaempf, Polymer Letters 14, 201-206 (1976); F. Lacoste, Doctorat ès Sciences, Université des Sciences et Techniques du Languedoc, Montpellier (1976); M. Viguier, A. Collet and F. Schué, J. Phys. Chem. 82, 1578-1579 (1978).

(45) B. Kaempf, S. Raynal, A. Collet, F. Schué, S. Boileau and J.M. Lehn, Angew. Chem. Internat. Edit. 86, 611-612 (1974).

(46) G.V. Nelson and A. von Zelevsky, J.Amer. Chem. Soc. 97, 6279-6281 (1975); G.F. Pedulli and A. Alberti, J.C.S. Perkin II 137-140 (197 $\overline{6)}$.

(47) J.L. Dye, J.M. Ceraso, M.T. Lok, B.L. Barnett and F.J. Tehan, J. Amer. Chem. Soc. 96, 608-609 (1974); J.L. Dye, C.W. Andrews and S.E. Mathews, J. Phys. Chem. 79, 30653070 (1975).

(48) F.J. Tehan, B.L. Barnett and J.L. Dye, J.Amer. Chem. Soc. 96, 7203-7208 (1974).

(49) J.L. Dye, M.R. Yemen, M.G. DaGue and J.M. Lehn, J. Chem. Phys. 68, 1165-1670 (1978); M.G. DaGue, J.S. Landers, H.L. Lewis and J.L. Dye, Chem. Phys. Letters 66, 169-172 (1979).

(50) D.G. Adolphson, J.D. Corbett and D.J. Merryman, J. Amer. Chem. Soc. 98, 7234-7239 (1976); A. Cisar and J.D. Corbett, Inorg. Chem. 16, 2482-2487 (1977); J.D. Corbett and P.A. Edwards, J. Amer. Chem. Soc. 99, 3313-3317 (1977); C.H.E. Belin, J.D. Corbett and A. Cisar, J. Amer. Chem. Soc. 99, 7163-7169 (1977) and references therein.

(51) B. Dietrich, J.M. Lehn and J.P. Sauvage, Tetrahedron Letters 2889-2892 (1969).

(52) M. Cinquini, F. Montanari and P. Tundo, J.C.S. Chem. Comm. 393-394 (1975); M. Cinquini, F. Montanari and P. Tundo, Gazetta Chim. Ital. 107, 11-14 (1977).

(53) D. Clément, F. Damm and J.M. Lehn, Heterocycles 5, 477-484 (1976).

(54) D. Landini, A. Maia, F. Montanari and P. Tundo, J. Amer. Chem. Soc. 101, 2526-2530 (1979); D. Landini, A. Maia and F. Montanari, J.C.S. Perkin II 46-51 (1980).

(55) D. Landini, F. Montanari and F. Rolla, Synthesis 223-225 (1978).

(56) D.J. Sam and H.E. Simmons, J. Amer. Chem. Soc. 96, 2252-2253 (1974) and footnote (6) therein.

(57) B. Dietrich and J.M. Lehn, Tetrahedron Letters 1225-1228 (1973) and unpublished results.

(58) D. Bauer and A. Caillet, Analusis 3, 440-442 (1975).

(59) J.L. Pierre, R. Le Goaller and H. Handel, J. Amer. Chem. Soc. 100, 8021-8022 (1978); J.L. Pierre, R. Le Goaller, H. Handel and A. Ripert, Tetrahedron Letters 3259-3262 (1978).

(60) R. Le Goaller, H. Handel, M.A. Pasquini and J.L. Pierre, Tetrahedron 35, 1437-1440 (1979).

(61) D. Clément and J.M. Lehn, unpublished observations.

(62) C.J. Pedersen, J. Amer. Chem. Soc. 89, 7017-7036 (1967).

(63) A. Schmidpeter and J.H. Weinmaier, Chem. Ber. 111, 2086-2098 (1978).

(64) S. Akabori and M. Ohtomi, BuZZ. Chem. Soc. Japan 48, 2991-2992 (1975).

(65) A. E1 Basyony, J. K1imes, A. Knöchel, J. Oehler and G. Rudolph, 2. Naturforsch. 31b, 1192-1200 (1976); W.L. Dorn, A. Knöche1, J. Oehler and G. Rudolph, Z. Naturforsch. 32b, 776-782 (1977); A. Knöche1, J. Oehler and G. Rudolph, Z. Naturforsch. 32b, 783-786 (1977).

(66) M. Gross and F. Peter, Bulz. Soc. Chim. France 871-874 (1975).

(67) S. Akabori and H. Tuji, Buzl. Chem. Soc. Japan 51, 1197-1199 (1978).

(68) J.F. Biellmann, H. D'Orchymont and M.P. Goeldner, Tetrahedron Letters 4209-4210 (1979).

(69) M. Cinquini, S. Colonna, H. Molinari, F. Montanari and P. Tundo, J.C.S. Chem. Comm. 394-396 (1976); F. Montanari and P. Tundo, Tetrahedron Letters 5055-5058 (1979).

(70) C. Cambillau, P. Sarthou and G. Bram, Tetrahedron Letters 281-284 (1976); C. Cambillau, G. Bram, J. Corset, C. Riche and C. Pascard-Billy, Tetrahedron 34, 2675-2685 (1978).

(71) J.L. Pierre and H. Handel, Tetrahedron Letters 2317-2320 (1974); J.L. Pierre, H. Handel and R. Perraud, Tetrahedron 31, 2795-2798 (1975); H. Handel and J.L. Pierre, Tetrahedron Letters 741-744 (1976).

(72) A. Loupy, J. Seyden-Penne and B. Tchoubar, Tetrahedron Letters 1677-1680 (1976).

(73) J.L. Pierre, H. Handel and R. Perraud, Tetrahedron Letters 2013-2016 (1977). 
(74) H. Handel and J.L. Pierre, Tetrahedron 31, 997-1000 (1975).

(75) H. Handel and J.L. Pierre, Tetrahedron 31, 2799-2802 (1975).

(76) A. Loupy and J. Seyden Penne, Tetrahedron Letters 2571-2574 (1978) and Tetrahedron, in press.

(77) S. Akabori and T. Yoshii, Tetrahedron Letters 4523-4526 (1978).

(78) A. Loupy, M.C. Roux-Schmitt and J. Seyden-Penne, unpublished results; private communication.

(79) C.J. Chang, R.F. Kiesel and T.E. Hogen-Esch, J. Amer. Chem. Soc. 95, 8446-8448 (1973).

(80) A. Deffieux and S. Boileau, Polymer 18,1047-1050 (1977); S. Boileau, A. Deffieux, D. Lasalle, F. Menezes and B. Vidal, Tetrahedron Letters 1767-1770 (1978).

(81) P.M. Atlani, J.F. Biellmann, S. Dube and J.J. Vicens, Tetrahedron Letters 2665-2668 (1974).

(82) J.F. Biellmann and J.L. Schmitt, Tetrahedron Letters 4615-4618 (1973).

(83) J.F. Biellmann and J.J. Vicens, Tetrahedron Letters 2915-2918 (1974).

(84) G. Chassaing and A. Marquet, Tetrahedron 34, 1399-1404 (1978).

(85) J. Smid, A.J. Varma and S.C. Shah, J. Amer. Chem. Soc. 101, 5764-5769 (1979).

(86) S. Boileau, B. Kaempf, J.M. Lehn and F. Schué, Polymer Letters 12, 203-209 (1974); S. Boileau, B. Kaempf, S. Raynal, J. Lacoste and F. Schué, Polymer Letters 12, 211216 (1974); S. Boileau, P. Hemery, B. Kaempf, F. Schué and M. Viguier, Polymer Letters 12, 217-220 (1974); P. Hemery, S. Boileau and P. Sigwalt, J. Polymer Sci. B 13, 49-54 (1975); M. Viguier, M. Abadie, F. Schué and B. Kaempf, Eur. Polymer J. 13, 213-222 (1977); S. Alev, A. Collet, M. Viguier and F. Schué, J. of Polymer Science 18, 11631169 (1980); A. Deffieux and S. Boileau, Macromolecules $\underline{9}$, 369-371 (1976).

(87) H. Dang Ngoc, Thèse de Doctorat ès Sciences, Université Pierre et Parie Curie, (1979)

(88) W. Edge11 and S. Chanjamsri, J. Amer. Chem. Soc. 102, 147-155 (1980). 